\title{
Molecular Motion of Long Deoxyribonucleic Acid Chains in a Concentrated Polymer Solution Depending on the Frequency of Alternating Electric Field
}

\author{
Masanori Ueda, ${ }^{\dagger}$ Kenichi YoshiKawa, ${ }^{*}$ and Masao DoI** \\ Institute of Scientific and Industrial Research, Osaka University, Osaka 567-0047, Japan \\ * School of Science, Kyoto University, Kyoto 606-8502, Japan \\ ** School of Engineering, Nagoya University, Nagoya 464-8601, Japan
}

(Received November 6, 1998)

\begin{abstract}
Through direct observation of long deoxyribonucleic acid (DNA) by means of fluorescence microscopy, we investigated the dynamics of individual DNA chains under alternating current electric fields in concentrated polyacrylamide solutions. By the study on the conformational change of the DNA in a wide range of frequencies, we have noticed the existence of four different regions depending on the frequency, as for the dynamic behavior of the DNA; i.e., linear motion, anti-resonance, stretching, and orienting. The four regions are characterized by the time dependence of the maximum length $R_{l}$ and the position of the center of mass $G_{x}$ of the DNA. In the linear motion region, $R_{l}$ is almost constant, and $G_{x}$ changes sinusoidally with time. In the anti-resonance region, both $R_{l}$ and $G_{x}$ change sinusoidally. In the stretching region, both $R_{l}$ and $G_{x}$ are almost constant. In the orienting region, both $R_{l}$ and $G_{x}$ are almost constant, but the DNA is not fully stretched. We observed that long DNA molecules with various lengths, ranging from $16 \mu \mathrm{m}$ to over $200 \mu \mathrm{m}$, are elongated in the stretching region, indicating that this stretching phenomenon would be independent of the length of DNA. By the observation of the stretching process, it was found that the asymmetric effect of the electric field is essential for the stretching. We explain such asymmetric effect by using simple tube model.

KEY WORDS Direct Observation / Stretching of Deoxyribonucleic Acid / Linear Conformation / Electrophoresis in Polymer Solutions / Entanglement Effect /
\end{abstract}

The dynamics of deoxyribonucleic acid (DNA) during electrophoresis is an important area that must be understood to develop the separation techniques. It is also an interesting problem in polymer science related to the dynamics of confined polymers. By means of the fluorescence microscopy based on the work of Morikawa et al., ${ }^{1}$ it is now possible to observe a single DNA during electrophoresis directly. Many researchers have studied the separation mechanism of long DNA in gel electrophoresis with this technique, and have revealed the characteristic oscillatory motion of long DNA under steady electric fields. ${ }^{2-6}$ Shi et al. observed similar oscillatory motion (which we call U-shape motion) also in linear polymer solutions above and below the entanglement limit. ${ }^{7}$

Recently we reported that in a highly concentrated polymer solution, long DNA chains migrate with linear conformation under direct current (DC) electric fields. ${ }^{8,9}$ Under the DC electric field, the maximum length $R_{l}$ of the linear conformation was much shorter than the contour length of the DNA. In the case of T4 DNA, the natural contour length is $55 \mu \mathrm{m}$ and the maximum length $R_{l}$ of the DNA in the linear conformation was found to be $c a .16 \mu \mathrm{m}$ in a $7 \%$ polyacrylamide (PA) solution under a DC field of $45 \mathrm{~V} \mathrm{~cm}^{-1}$. We call this migrating pattern I-shape motion. On the other hand, under alternating current (AC) electric fields, we discovered that the fully stretched state of T4 DNA was attained under a proper condition. ${ }^{10.11}$ Figure 1 shows these three states of T4 DNA in a 7\% PA solution. Without the external field, DNA is in a random coil state (Figure 1a). Under steady electric fields, DNA migrates with linear conformation
(Figure 1b). However, DNA is never stretched up to the full contour length under any field strength. Under AC electric fields, DNA is in the almost fully stretched state as shown in Figure 1c. Although this stretching phenomenon is interesting, the mechanism was poorly understood in our previous work. ${ }^{10}$

In this article, we report more detailed features of the dynamics of a long DNA chain under AC electric fields in concentrated polymer solutions. Especially, the stretching behavior of DNA was investigated with a wide range of frequencies of electric field $(0.1-100 \mathrm{~Hz})$. Linear PA was used as a host polymer, being soluble in aqueous environment. For linear double-stranded DNA of various sizes $(48.5 \mathrm{kbp}, 166 \mathrm{kbp}, c a .1 \mathrm{Mbp})$, the stretching behavior were examined from the direct observation of individual DNA molecules using the fluorescence microscopy. In order to understand the stretching mechanism, we observed the stretching process of T4 DNA. Based on this observation, we advance the model used in our previous work. ${ }^{10}$

\section{EXPERIMENTAL}

\section{Host Polymer}

Linear PA $\left(\mathrm{MW}=7 \times 10^{5}-10 \times 10^{5}, 10 \%\right.$ in water, Tokyo Chemical Industry Co., Ltd.) was diluted with a TBE buffer solution (the final concentration: $45 \mathrm{mM}$ Tris-borate, $1.25 \mathrm{mM}$ EDTA, $\mathrm{pH} 8.3$ ). The contour length of the PA is of the order of $1 \mu \mathrm{m}$, which implies that the PA has enough length to entangle with a DNA chain. The entanglement limit $\mathrm{C}^{*}$ (in $0.5 \mathrm{TBE}$ buffer) of the PA was estimated to be $c a$. $0.7 \%$ from viscosity

\footnotetext{
† Correspondence should be addressed to the author M.U. Present address: Department of Medicinal Chemistry, Faculty of Pharmaceutical Sciences,
} The University of Tokushima, Shomachi, Tokushima 770-8505, Japan (e-mail: uedam@fc.ph.tokushima-u.ac.jp). 
a)

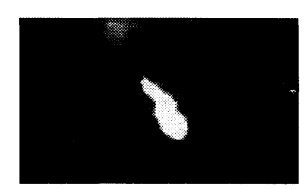

b)

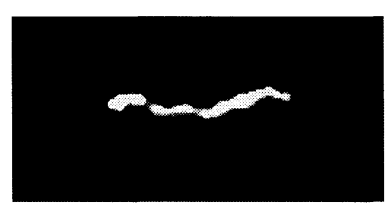

c)

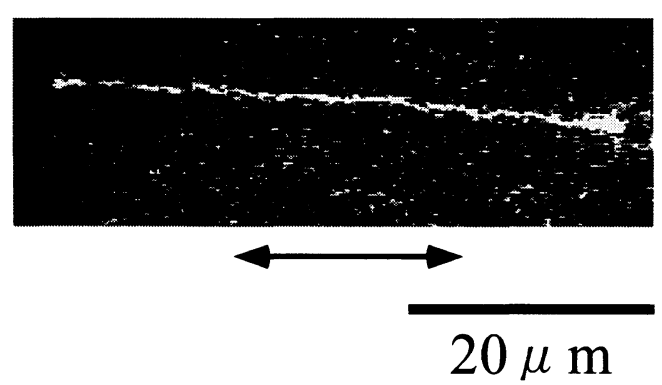

Figure 1. Fluorescence images of T4 DNA in $7 \%(w / w)$ linear polyacrylamide solution. a) Random coil state of DNA (double stranded) in the absent of external electric field. b) Linear conformation of DNA under a DC electric field $\left(10 \mathrm{~V} \mathrm{~cm}^{-1}\right)$. DNA migrates in the direction of the inserted arrow. c) Stretched state of DNA under an AC electric field $\left(200 \mathrm{~V}_{\mathrm{pp}}, 6 \mathrm{~Hz}\right)$. The electric field was applied in the horizontal direction.

measurement.

\section{$D N A$}

$\lambda$ DNA (48.5 kbp, Takara) and T4 DNA (166 kbp, Nippon Gene), stained with a fluorescence dye, YOYO-1 (Molecular Probes, Inc.), were mixed with a PA solution (in $0.5 \mathrm{TBE}$ buffer) including $4 \%(\mathrm{v} / \mathrm{v}) 2$-mercaptoethanol, $2.3 \mathrm{mg} \mathrm{ml}^{-1}$ glucose, $0.1 \mathrm{mg} \mathrm{ml}^{-1}$ glucose oxidase, and $0.018 \mathrm{mg} \mathrm{ml}^{-1}$ catalase. These solutions, including either $\lambda \mathrm{DNA}$ or T4 DNA, were injected between two glass plates. Other details of the sample preparations are similar to those used in the previous work. ${ }^{10}$

Saccharomyces cerevisiae chromosomal DNA (ca. $200 \mathrm{kbp}-2 \mathrm{Mbp}$, Bio Rad) was separated by $120^{\circ}$ pulsedfield gel electrophoresis. After separation, the bands of $1.10 \mathrm{Mbp}$ and $1.12 \mathrm{Mbp}$ were cut with a razor blade and placed into a microtube with $\beta$-agarase buffer $(40 \mathrm{mM}$ Bis-Tris/ $\mathrm{HCl}, 40 \mathrm{mM} \mathrm{NaCl}, 1 \mathrm{mM}$ EDTA (pH 6.0)) for $60 \mathrm{~min}$ at room temperature. Then, the buffer was discarded. The gel slice thus recovered was allowed to melt at $70^{\circ} \mathrm{C}$ for $15 \mathrm{~min}$ and cooled to $45^{\circ} \mathrm{C}$. Then, $\beta$-agarase (FMC) was added to it. The mixture was incubated at $45^{\circ} \mathrm{C}$ for $60 \mathrm{~min}$. After this incubation, an excess solution was discarded, and YO-PRO (Molecular Probe, Inc.) was added. The mixture was stored at $4^{\circ} \mathrm{C}$ for $24 \mathrm{~h}$. Finally, PA solution and other chemical components were added to the solution containing the DNA and dye.

The electrophoretic miniature cell on the microscope was used for direct observation. Two Pt electrodes were placed with the distance of $c a .1 \mathrm{~cm}$ in the miniature

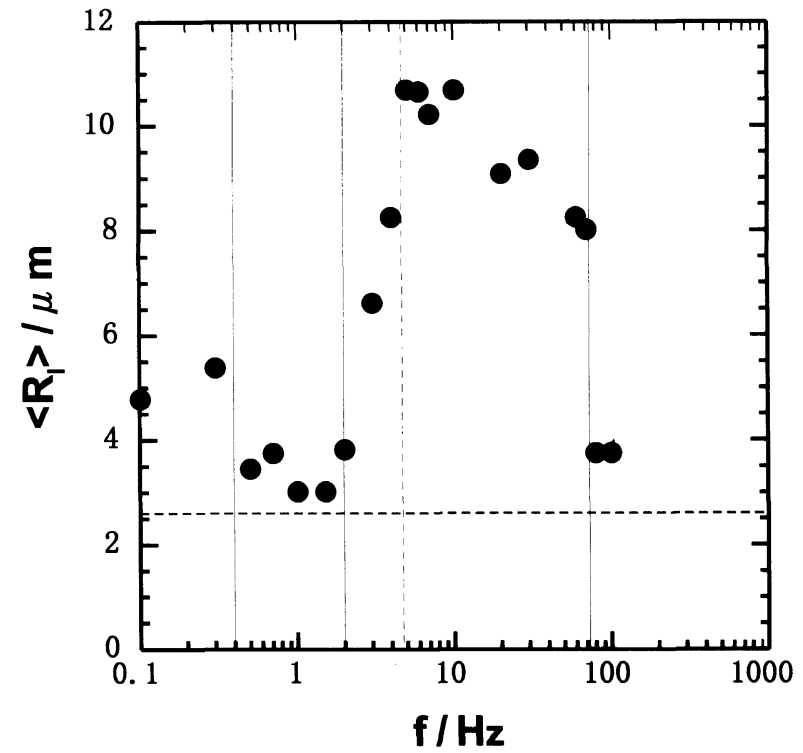

Figure 2. Average maximum length $\left\langle R_{l}\right\rangle$ for $\lambda$ DNA as a function of the electric field frequency $f$. Maximum length $R_{l}$ of each molecule was averaged for $10-20 \mathrm{~s}$ for more than 5 different molecules. Horizontal dashed line indicates the equilibrium value of $\lambda$ DNA in a random coil state. Vertical solid lines divide frequency into four regions, linear motion (less than $0.4 \mathrm{~Hz}$ ), anti-resonance (from $0.4 \mathrm{~Hz}$ to $2 \mathrm{~Hz}$ ), stretching $(2-75 \mathrm{~Hz})$, and orienting (more than $75 \mathrm{~Hz})$. Vertical dashed line indicates the transition region $(2-4 \mathrm{~Hz})$ from anti-resonance to stretching.

cell. Sinusoidal electric fields of $200 \mathrm{~V}_{\mathrm{pp}}$ (peak-topeak voltage) were applied between the electrodes. The microscope and other experimental apparatus were similar to those used in the previous work..$^{9,10}$ The images of Figures 1a, 1b, 1c, 3a, 4a, 5a, 6a, 7a, and 8 were processed with Photoshop (Adobe Systems, Inc.). The gray level and the contrast were optimized for each figure.

\section{RESULTS}

\section{Dependence of Maximum Length on Frequency}

We used $\lambda$ DNA to measure the average maximum length $\left\langle R_{l}\right\rangle$ as a function of the electric field frequency. For clear observation of the effect of the frequency on $\left\langle R_{l}\right\rangle$, we chose $\lambda$ DNA instead of T4 DNA because the T4 DNA molecules are occasionally trapped in a hairpin shape for long time before exhibiting a completely elongated conformation. In order to avoid this trapping, it is necessary to disturb the hairpin structure by an electric field with low frequencies (about $0.1 \mathrm{~Hz}$ ). ${ }^{10} \mathrm{On}$ the other hand, $\lambda \mathrm{DNA}$ are rarely trapped in the hairpin state and this low-frequency treatment is not necessary.

Figure 2 shows the average maximum length $\left\langle R_{l}\right\rangle$ as a function of the frequency of the electric field. The maximum length $R_{l}$ of each molecule was averaged for $10-20 \mathrm{~s}$ for more than 5 different molecules. Horizontal dashed line indicates the equilibrium maximum length of $\lambda \mathrm{DNA}$ in a random coil state. We classified the frequency into the following four regions as indicated by the vertical solid lines in Figure 2.

Linear Motion (less than ca. $0.4 \mathrm{~Hz}$ ). In this region, DNA exhibits a simple linear motion as shown in Figures $3 \mathrm{a}$ and $3 \mathrm{~b}$. During migration, $R_{l}$ remains almost constant 
a)

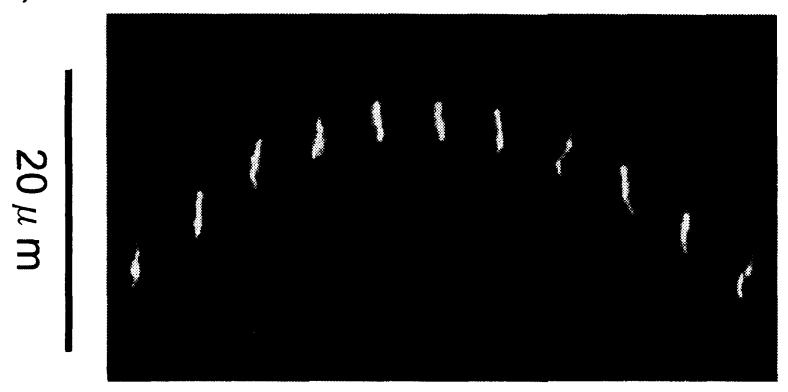

b)

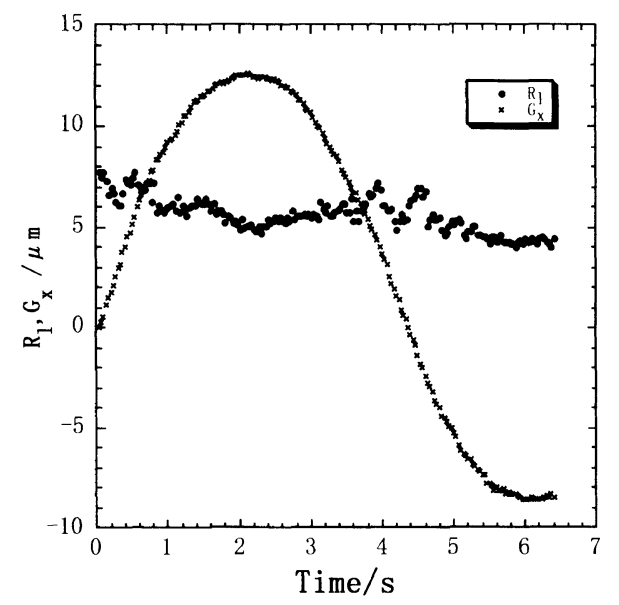

Figure 3. Region for linear motion. a) Fluorescence images of $\lambda$ DNA during linear motion. Electric field with the frequency of $0.1 \mathrm{~Hz}$ was applied in the vertical direction. Each image was taken with the interval of $0.5 \mathrm{~s}$ from left to right. b) Time evolution of the maximum length $R_{l}(\bigcirc)$ and the center of mass $G_{x}(\times)$ of the $\lambda$ DNA during linear motion. $R_{l}$ keeps almost constant value, $c a$. $6 \mu \mathrm{m}$, during migration. On the other hand, the trajectory of the center of mass $G_{x}$ is sinusoidal.

at $c a .6 \mu \mathrm{m}$. Notice that this $R_{l}$ is less than the natural contour length (ca. $16 \mu \mathrm{m})$. On the other hand, the position of the center of mass $G_{x}$ changes almost sinusoidally with time. In this region, the motion of DNA is essentially the same as that of the linear motion under DC electric fields.

Anti-Resonance (from $0.4 \mathrm{~Hz}$ to $2 \mathrm{~Hz}$ ). In this region, $R_{l}$ is in a range between those for the random coil and linear conformation states. Both $R_{l}$ and $G_{x}$ exhibit a sinusoidal response for external field as shown in Figures $4 \mathrm{a}$ and $4 \mathrm{~b}$. We call this region as anti-resonance region, following the naming in the current studies on the field inversion ${ }^{12}$ or the biased sinusoidal ${ }^{13}$ gel electrophoresis.

Stretching (from $2 \mathrm{~Hz}$ to $75 \mathrm{~Hz}$ ). In this region, DNA is almost fully elongated (except at its ends). Both $R_{l}$ and $G_{x}$ have almost constant values as shown in Figures $5 \mathrm{a}$ and $5 \mathrm{~b}$. The region between $2 \mathrm{~Hz}$ and $4 \mathrm{~Hz}$ is a transition region from anti-resonance to stretching. At $2 \mathrm{~Hz}, 3 \mathrm{~Hz}$, and $4 \mathrm{~Hz}$, DNA shows oscillatory motion with elongated conformation. However, the oscillation is not synchronized with the external field. On the other hand, above $20 \mathrm{~Hz}$, DNA takes a slightly relaxed conformation. Consequently, in this stretching region, the average maximum length $\left\langle R_{l}\right\rangle$ has one peak value around $10 \mathrm{~Hz}$ as shown in Figure 2.

Orienting (over $75 \mathrm{~Hz}$ ). In this region, DNA is not a)

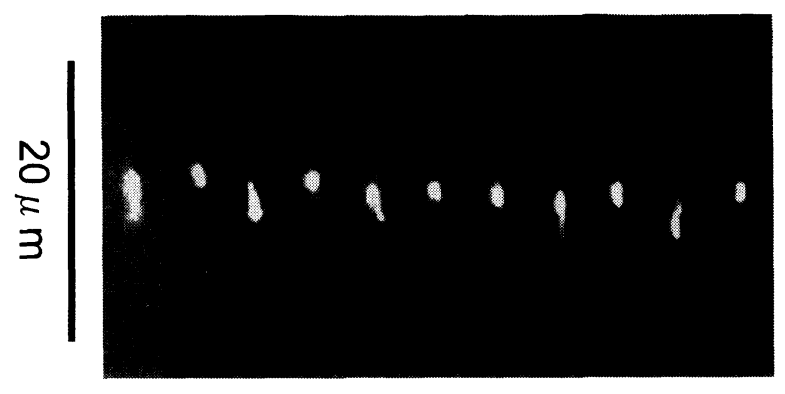

b)

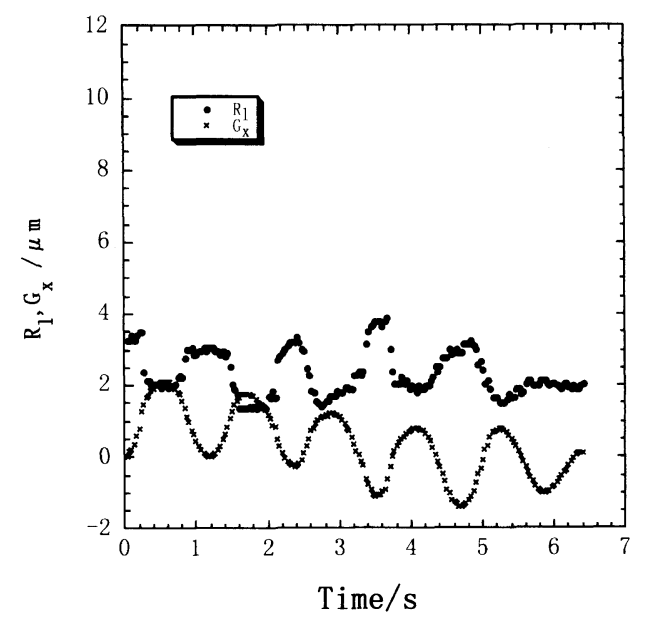

Figure 4. Region for anti-resonance. a) Fluorescence images of $\lambda$ DNA during anti-resonance motion. Frequency is $1 \mathrm{~Hz}$, and other conditions are same to Figure 3. b) Time evolution of the maximum length $R_{l}(\bigcirc)$ and the center of mass $G_{x}(\times)$. Both $R_{l}$ and $G_{x}$ have a sinusoidal response for the external field.

elongated but orienting in the direction of the external field. Both $R_{l}$ and $G_{x}$ have almost constant values as shown in Figures $6 \mathrm{a}$ and $6 \mathrm{~b} . R_{l}$ takes a value between those for the random coil and fully elongated states.

\section{Stretching Process}

Figure 7a shows the process of stretching of T4 DNA in $7 \%$ PA. Here, DNA was elongated beforehand by shear flow (in the direction of white arrow shown in the first picture of Figure 7a). An electric field with the frequency of $10 \mathrm{~Hz}$ was applied in the horizontal direction. Each image was taken with the interval of $2 \mathrm{~s}$. In the figure, it is to be noted that two major kinks are growing in the opposite directions. This means that under electric fields around $10 \mathrm{~Hz}$, only the kinks in the random coiled DNA can break the "tube" formed by host polymers. Time evolution of the distance between the two kinks $\left(L_{\mathrm{kk}}\right)$ is plotted in Figure $7 \mathrm{~b}$, where the slope of the fitted line is $0.96 \mu \mathrm{m} \mathrm{s}^{-1}$. Supposing that the two kinks move with the same speed in the opposite direction, the speed is evaluated to be $0.48 \mu \mathrm{m} \mathrm{s}^{-1}$.

We observed that DNA stretches also under an alternating square pulse field. However, the DNA ceases to stretch if the shape of the pulse field becomes asymmetric. We have not yet ascertained whether the critical value of the asymmetric parameter exists or 
a)

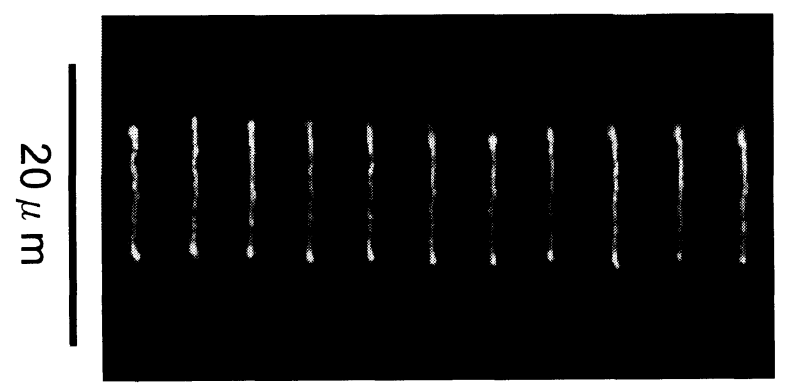

b)

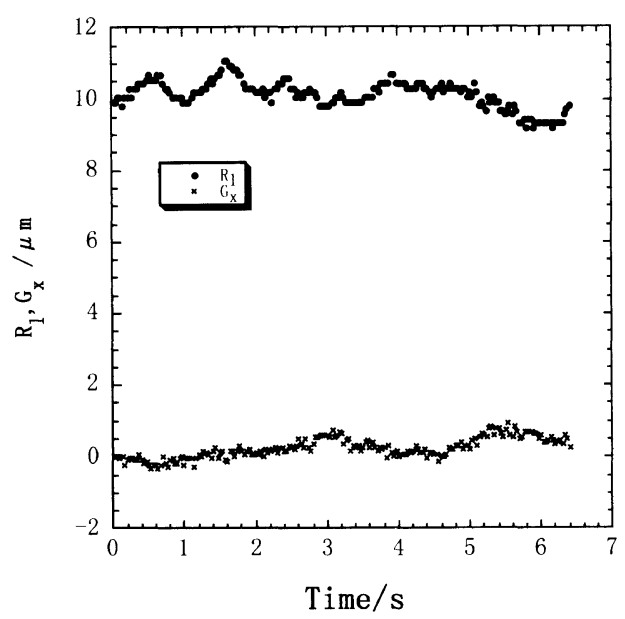

Figure 5. Region for stretching. a) Fluorescence images of $\lambda$ DNA in an elongated state. DNA is fully stretched, except for the end. Frequency is $10 \mathrm{~Hz}$, and other conditions are same to Figure 3. b) Time evolution of the maximum length $R_{l}(\bigcirc)$ and the center of mass $G_{x}$ (×). Both $R_{l}$ and $G_{x}$ have almost constant values.

not.

\section{Stretching of Mbp-Sized DNA}

Figure 8a shows that Saccharomyces cerevisiae chromosomal DNA (ca. $1 \mathrm{Mbp}$ ) is almost fully stretched under an electric field at $5 \mathrm{~Hz}$ in $8 \%$ PA. Figure 8 b shows the trace of the fluorescence image in Figure 8a. Although the observed maximum length $R_{l}$ of this DNA is $c a$. $200 \mu \mathrm{m}$, its natural contour length calculated from the number of the base pairs is over $300 \mu \mathrm{m}$. The DNA might have been cut on the pipetting operation from the micro-tube to the electrophoretic miniature cell. Therefore, we have conjectured that even Mbp-sized DNA takes the stretched conformation with the AC field. Moreover, it should be noted that DNA molecules with a wide range of lengths (from $c a .10 \mu \mathrm{m}$ to $c a .200 \mu \mathrm{m}$ ) are stretched under almost the same conditions (about $10 \mathrm{~Hz}$, in $7-8 \%$ PA solution). Strictly speaking, as shown in Figure 8b, Mbp-sized DNA molecules always have big burls in the stretched state and cannot attain to the completely stretched state.

\section{DISCUSSION}

In this section, we semi-quantitatively describe the a)

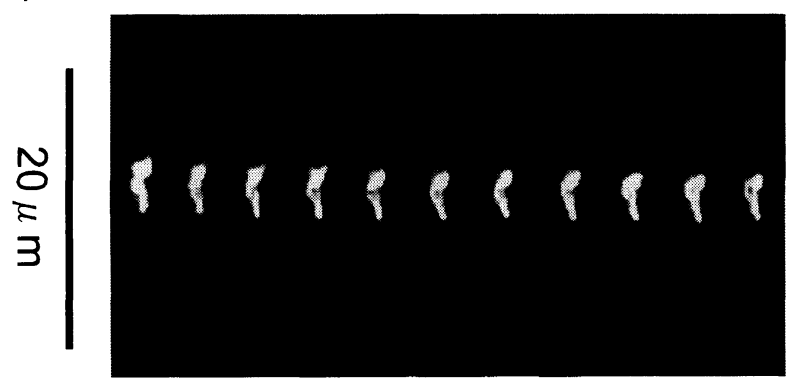

b)

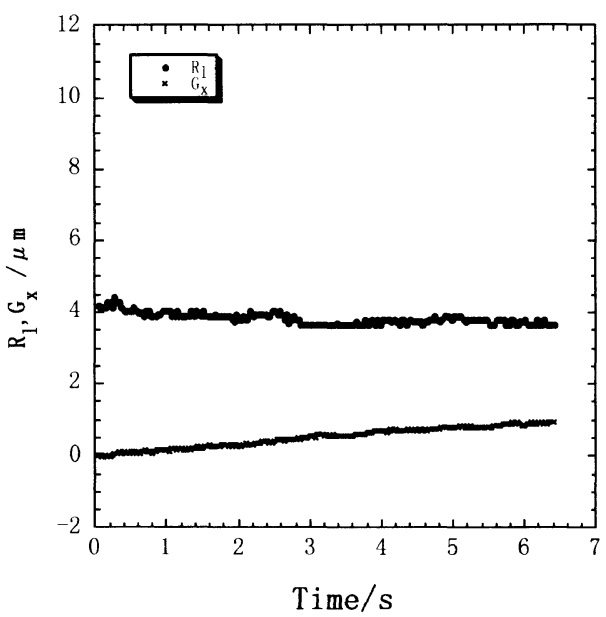

Figure 6. Region for orienting. a) Fluorescence images of iDNA in an orienting and not elongated state. Frequency is $100 \mathrm{~Hz}$, and other conditions are same to Figure 3 . b) Time evolution of the maximum length $R_{l}(\bigcirc)$ and the center of mass $G_{x}(\times)$. Both $R_{l}$ and $G_{x}$ have almost constant values. $R_{l}$ is larger than that in the random coil state, but is shorter than that in the stretched state. DNA is not elongated but orienting in the direction of the external field.

results mentioned in the preceding section, and propose the mechanism of the stretching.

\section{Electrophoretic Distance and Velocity of Long DNA in Each Region}

In order to understand the mechanism of the transition behavior, it is useful to study the electrophoretic distances $\left(D_{\mathrm{el}}\right)$ of the DNA segments at each frequency. At $0.1 \mathrm{~Hz}$, the peak-to-peak value of $G_{x}$ during a half period $(5 \mathrm{~s})$ in Figure $3 \mathrm{~b}$ gives $D_{\mathrm{el}} \approx c a .20 \mu \mathrm{m}$. The average velocity $\left(V_{\mathrm{DNA}}\right)$ is, $20 \mu \mathrm{m} / 5 \mathrm{~s}=4 \mu \mathrm{m} \mathrm{s}^{-1}$. This estimation of the averaged velocity is ascertained from Figure $4 \mathrm{~b}$, in which $D_{\mathrm{el}}$ is $c a .2 \mu \mathrm{m}$, and the average velocity is $c a .4 \mu \mathrm{m} \mathrm{s}^{-1}$. These results indicate that the average velocity is independent of the frequency. Although we cannot evaluate $D_{\mathrm{el}}$ directly from the data of Figures $5 \mathrm{~b}$ and $6 \mathrm{~b}$, $D_{\text {el }}$ are estimated to be $0.2 \mu \mathrm{m}$ and $0.02 \mu \mathrm{m}$, respectively, if we assume that the average velocity remains constant.

\section{Transition Behavior of Long DNA Depending on Field Frequency}

We first consider the transition from the linear motion to the anti-resonance motion observed at low frequencies. It may be interpreted as follows. When an electric field is applied on DNA in a polymer solution, initially the 
a)
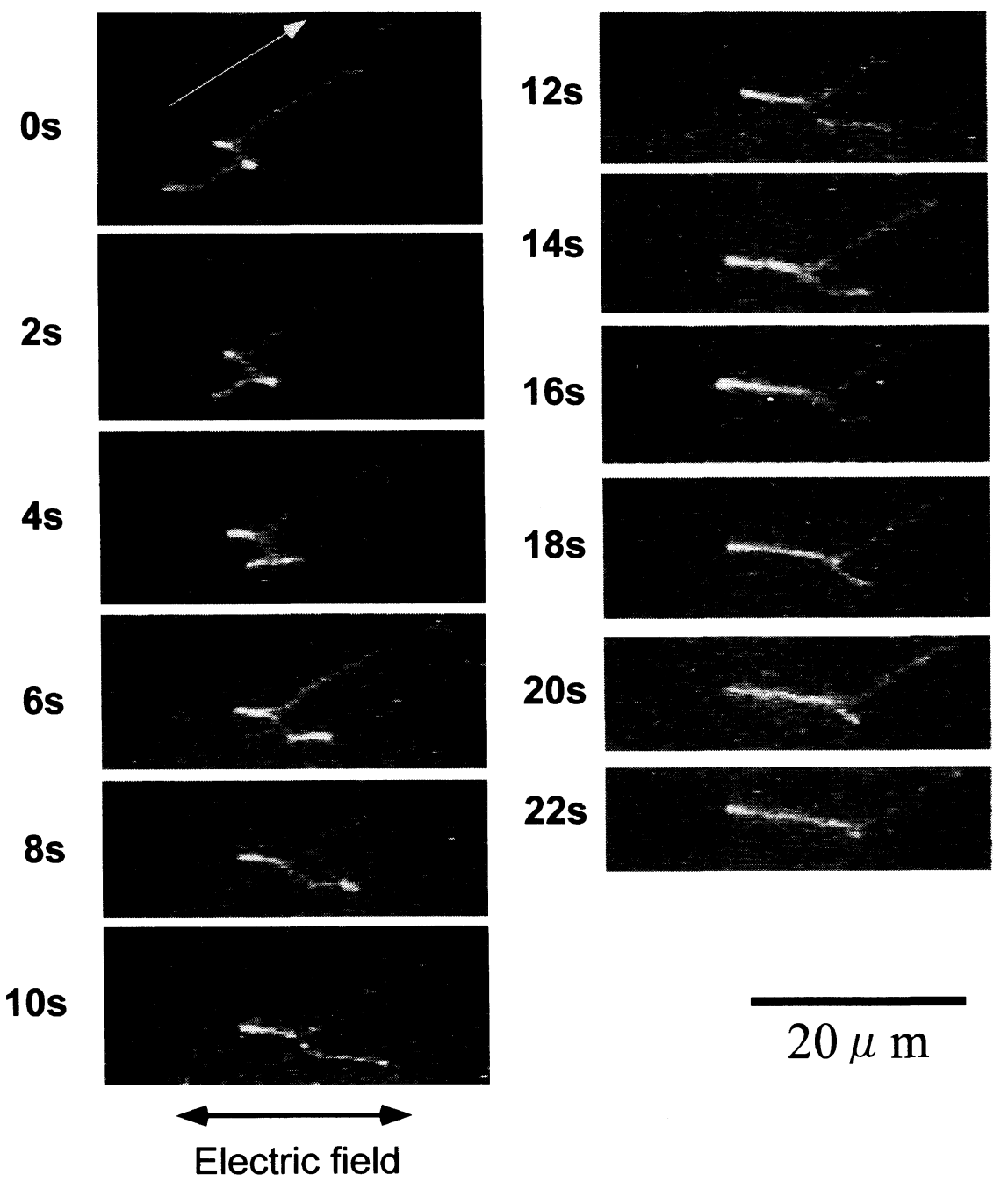

b)

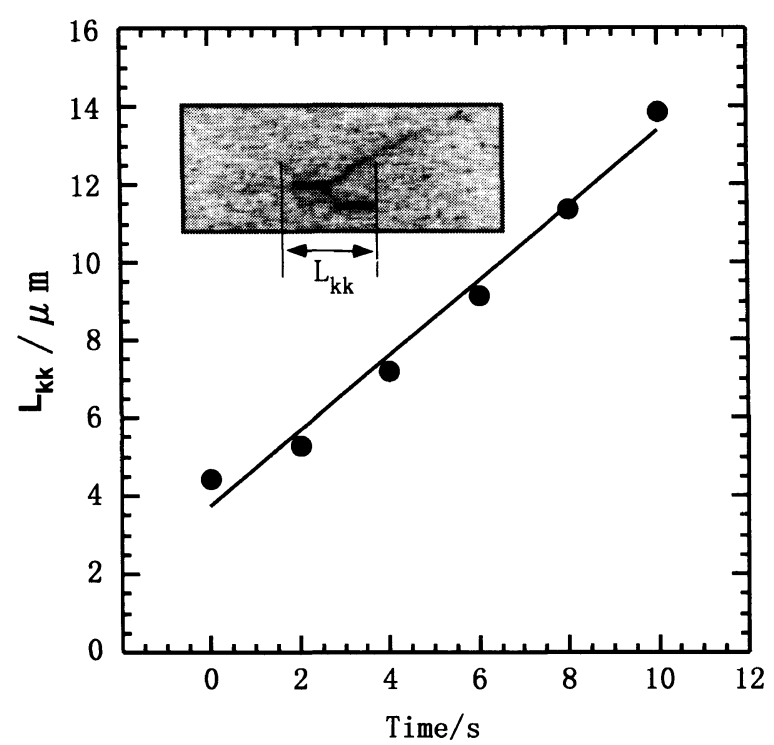

Figure 7. Stretching process of T4 DNA. In order to observe the initial stretching process, DNA was beforehand elongated by shear flow in $7 \%$ PA. a) Sequential pictures of the stretching process. Shear flow was applied in the direction of the white arrow inserted in the first picture. An electric field of $10 \mathrm{~Hz}$ was applied in horizontal direction. Each image was taken with the interval of $2 \mathrm{~s}$. Two major kinks move in the opposite direction. b) Time evolution of distance between the two kinks $\left(L_{\mathbf{k k}}\right)$. The slope of the fitted line is $0.96 \mu \mathrm{m} \mathrm{s}^{-1}$. 


\section{a)}

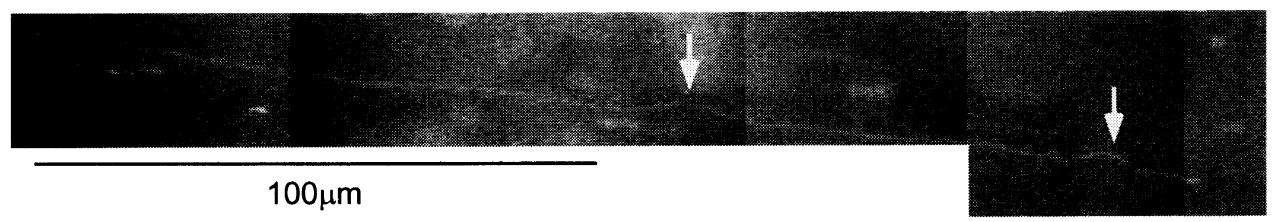

b)

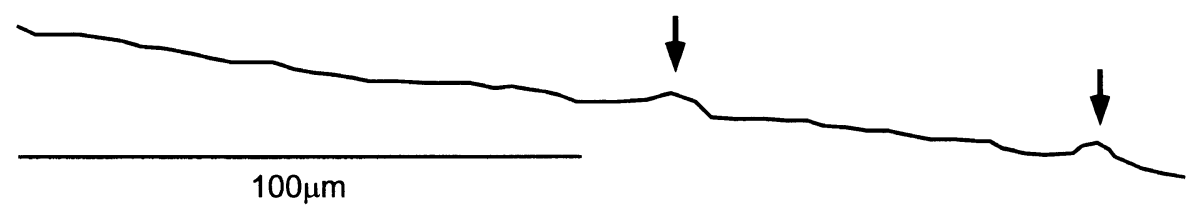

Figure 8. Stretching of Mbp-sized DNA. a) Saccharomyces cerevisiae chromosomal DNA (1.1 Mb, $1.12 \mathrm{Mb}$ ) separated by pulsed-field gel electrophoresis is almost fully stretched under the electric field with $5 \mathrm{~Hz}$ frequency in $8 \%$ PA. In order to get the fully stretched state, the frequency of the field was gradually changed from $0.1 \mathrm{~Hz}$ to $5 \mathrm{~Hz}$. Arrows indicate burls in the stretched DNA. b) Schematic trace of the above fluorescence image of the stretched Saccharomyces cerevisiae chromosomal DNA. Arrows indicate burls in the stretched DNA. Mbp-sized DNA molecules always have such burls in the stretched state.

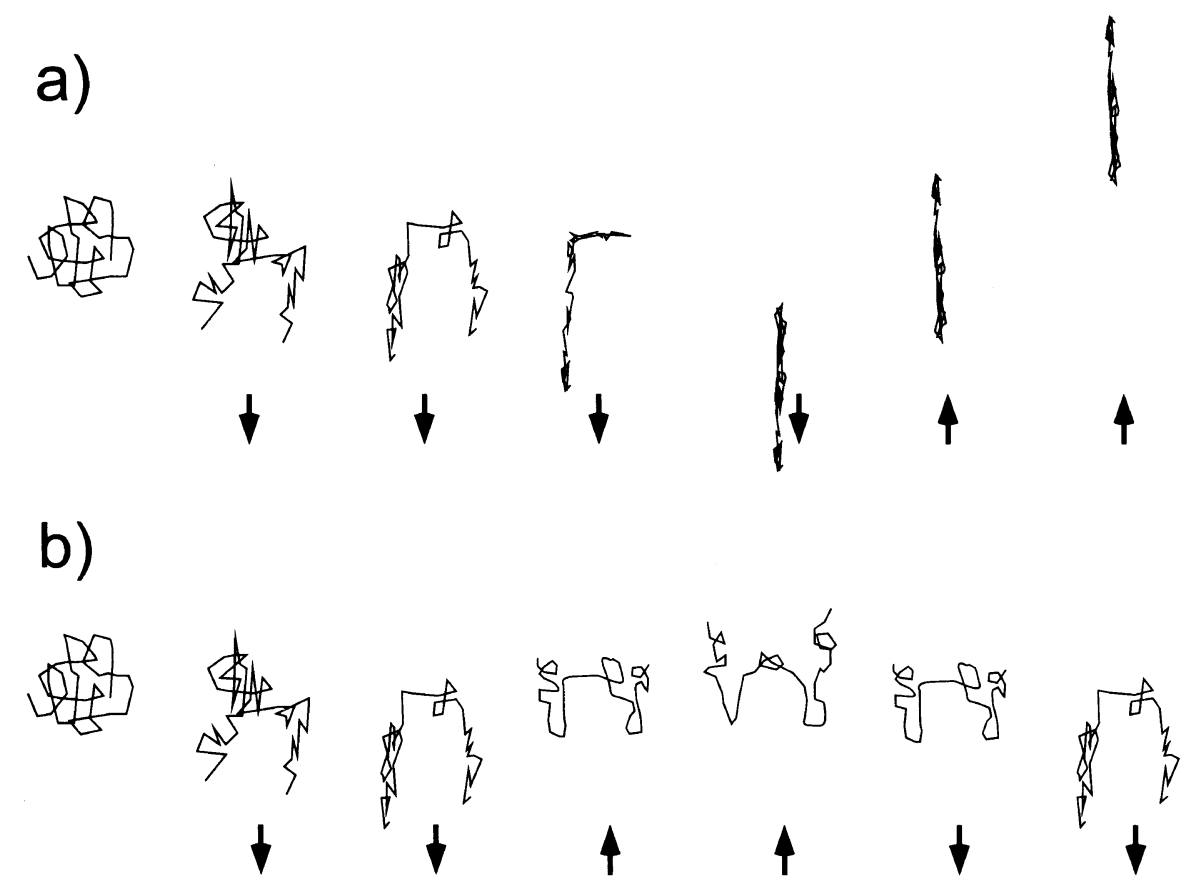

Figure 9. Schematic diagram of two different responses of a long DNA depending on the frequency. Vertical arrows indicate the direction of electric force. a) Region of linear motion: the half period of the external field is longer than the escape time $\left(\tau_{\text {DNA }}\right)$ of a U-shaped DNA around an obstacle. b) Region of anti-resonance: the half period of the external field is shorter than the escape time $\left(\tau_{\mathrm{DNA}}\right)$ of a U-shaped DNA around an obstacle. The direction of electric field is changing during the competition of two arms of the U-shaped DNA, and the two arms are forming smaller U-shape in the original U-shape.

DNA forms a U-shape. The escape time $\left(\tau_{\mathrm{DNA}}\right)$ of the $\mathrm{U}$-shaped DNA around an obstacle is estimated to be $\tau_{\text {DNA }} \sim L_{\text {DNA }} / V_{\text {DNA }}$, where $L_{\text {DNA }}$ is the contour length of the DNA, and $V_{\mathrm{DNA}}$ is the mean velocity of the DNA. ${ }^{14}$ If the half period of the external field is longer than $\tau_{\mathrm{DNA}}$, DNA can escape from the obstacle and move, keeping the linear conformation as shown in Figures $3 a, 3 b$, and $9 \mathrm{a}$. On the other hand, if the half period of the external field is shorter than $\tau_{\text {DNA }}$, DNA cannot escape from the obstacle. As the result, the DNA remains in the antiresonance state. When the field is inverted in the intermediate state to form U-shape, other small apexes are created in the original U-shape as shown in Figure $9 \mathrm{~b}$, because both ends of long DNA can move faster than the apex of the U-shape. ${ }^{9}$ Then, the DNA remaining in the anti-resonance state has many kinks in the single chain, and the kinks have apexes with opposite directions. However, we can hardly observe the resulting small kinks because of the blurring effect of fluorescence.

For $\lambda \mathrm{DNA}, L_{\mathrm{DNA}}$ is $16 \mu \mathrm{m}$, and $V_{\mathrm{DNA}}$ is estimated to be $4 \mu \mathrm{m} \mathrm{s}^{-1}$. Escape time $\tau_{\mathrm{DNA}}$ is calculated to be $16 \mu \mathrm{m} / 4 \mu \mathrm{m} / \mathrm{s}=4 \mathrm{~s}$. Therefore, the transition frequency is estimated to be $1 / 2 \times 4 \mathrm{~s}=0.125 \mathrm{~Hz}$. The observed transition frequency is $c a$. $0.4 \mathrm{~Hz}$, as shown in Figure 2 . 
It should be noted that this transition from the linear motion to the anti-resonance motion is essentially the same as that found in the field inversion ${ }^{12}$ and the biased sinusoida $1^{13}$ gel electrophoresis experiments. Therefore, this anti-resonance region may disappear for short DNA as noted in those experiments.

The second transition, from the anti-resonance to the stretching, occurs at intermediate frequencies. This transition is discussed in the following sections in relation to the stretching mechanism.

Finally, we consider the third transition, that is, from the stretching region to the orienting region. Figures $6 \mathrm{a}$ and $6 \mathrm{~b}$ show that the DNA is orienting but not stretching in this region. When the frequency is changed from $10 \mathrm{~Hz}$ to $100 \mathrm{~Hz}$ after stretching of the DNA at $10 \mathrm{~Hz}$, the DNA starts to relax. However, in this region, the DNA needs very long time to reach a steady state. In order to understand the behavior of DNA in this region, further investigation for the initial stage of orientation is necessary.

\section{Conditions of Electrophoretic Distance for DNA-Stretch- ing}

For DNA to be stretched in the concentrated polymer solutions, the electrophoretic distance of the DNA segment $\left(D_{\mathrm{el}}\right)$ must be adjusted to around $0.2 \mu \mathrm{m}$ by the external field. As the persistent length of double-stranded DNA $\left(\lambda_{\text {per }}\right)$ is $c a .0 .06 \mu \mathrm{m},{ }^{15} \lambda_{\text {per }}$ is less than $D_{\mathrm{el}}$ in the stretching region. This fact suggests that the motion of the partial chain longer than $\lambda_{\text {per }}$ plays an important role in the stretching of the whole chain.

The radius of gyration $\left(R_{\mathrm{g}}\right)$ of $\lambda \mathrm{DNA}$ in the random coil state is estimated to be $c a$. $1 \mu \mathrm{m}$ from the observed maximum length of $\lambda$ DNA in Figure 2 . When $D_{\mathrm{el}}$ satisfies the condition, $\lambda_{\text {per }}<D_{\mathrm{el}}<R_{\mathrm{g}}$, $\lambda \mathrm{DNA}$ will be stretched. However, the process of DNA-stretching observed in Figure $7 \mathrm{a}$ shows that dynamics of the kinks of the DNA plays an important role. In order to maintain the kinks, the $R_{\mathrm{g}}$ of the DNA must be larger than the size of the kinks. We speculate that the critical kink size $\left(L_{\mathrm{cr}}\right)$ exists for the stretching, and $\lambda_{\mathrm{per}}<D_{\mathrm{el}}<L_{\mathrm{cr}}$ is the condition for the DNA-stretching. Moreover, we can make a conjecture that the mechanism of stretching would be independent of the contour length of DNA if the size of DNA satisfies the condition, $L_{\mathrm{cr}}<R_{\mathrm{g}}$. In fact, DNA chains with various lengths (ca. $10-200 \mu \mathrm{m})$ are stretched under almost the same conditions (7-8\% PA, $5-10 \mathrm{~Hz})$. On the other hand, the DNA shorter than $\lambda$ DNA could not be stretched under the same condition (data not shown). In the future work, systematic studies on DNA length are required to investigate the critical kink size $\left(L_{\mathrm{cr}}\right)$ experimentally.

\section{Simple Explanation for the Mechanism of DNA-Stretch- ing}

Now we give a simple explanation for the stretching mechanism. In our previous work, ${ }^{10}$ we used the tube model to explain the asymmetric effect on the kinks of DNA. Here, we advance the model to explain the observations of the characteristic stretching process.

As shown in Figure 7a, the apex of the kink can move only in the direction that the apex points to. This observation is explained in the following way. When an
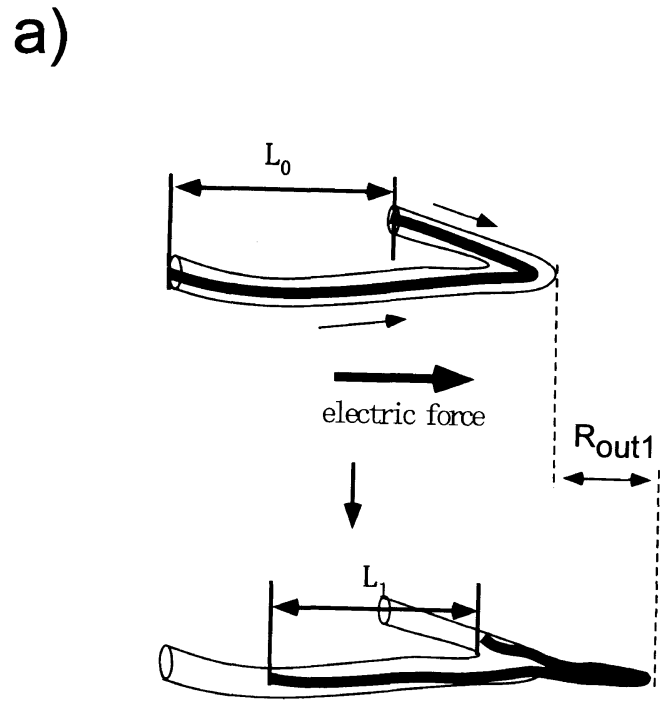

\section{b)}

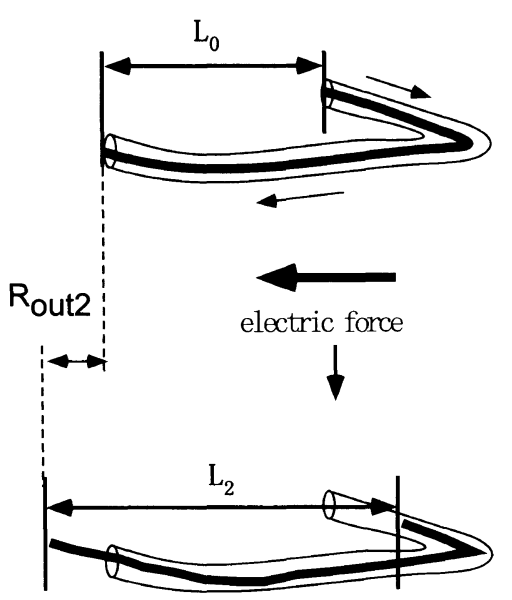

Figure 10. Schematic diagram of a kink of DNA in the tube of host polymers. As the electric field is symmetric, the effect on the kink of DNA is asymmetric. The reason for the assumption of asymmetry is based on the observation of stretching process, that is, the apex of the kink can only move in the direction that the apex points to. a) Direction of electric force is same as the apex of kink. Both shorter and longer arms move in tube in the direction of the electric force. Then, the apex breaks the old tube by the distance $R_{\text {out } 1}$, however, the end-to-end distance $L_{1}$ (after applying electric field) is nearly equal to $L_{0}$ (before applying electric field). b) Direction of electric force is opposite to the apex of kink. The longer arm pulls up the shorter arm. Then, the longer arm escapes from the old tube by the distance $R_{\text {out } 1}$. The end-to-end distance $L_{2}$ (after applying electric field) is larger than $L_{0}$ (before applying electric field).

electric force is applied in the same direction to the apex as shown in Figure 10a, both shorter and longer arms move in the direction of the electric force. Then, the apex moves out of the old tube by the distance $R_{\text {out } 1}$. However, the end-to-end distance $L_{1}$ after the application of the electric field is nearly equal to $L_{0}$ that before the application of the electric field. On the other hand, when an electric force is applied in the opposite direction to the apex as shown in Figure 10b, the longer arm pulls up the shorter arm. Then, the longer arm escapes from 
the old tube by the distance $R_{\text {out } 2 \text {. The apex is held at }}$ the same position. The end-to-end distance $L_{2}$ after the application of the electric field is larger than $L_{0}$. Therefore, the effect of symmetric external field on the kink of DNA should be asymmetric.

Here, we try to estimate the value of $R_{\text {out } 1}$ from the observation in Figures $7 \mathrm{a}$ and $7 \mathrm{~b}$. In the case of $10 \mathrm{~Hz}$, the moving speed of one kink calculated from Figures $7 \mathrm{a}$ and $7 \mathrm{~b}$ is $0.48 \mu \mathrm{m} \mathrm{s}^{-1}$. Consequently, $R_{\text {out } 1}$ is estimated to be $0.048 \mu \mathrm{m}$. This value is less than the electrophoretic distance of the DNA segment, $0.2 \mu \mathrm{m}$ estimated from Figures $3 \mathrm{~b}$ and $4 \mathrm{~b}$. This fact suggests that the movement of the kink is not completely asymmetric. If we perform the experiment in a less concentrated PA solution, the electrophoretic distance should become larger, and $R_{\text {out } 1}$ should become smaller. This may be attributed to that the apex of the kink moves equally in both directions of the electric field in that solution including a diffuse PA mesh. Therefore, it is expected that the asymmetric effect of external field will disappear on a decrease of PA concentration. In fact, we have never observed the stretching in semi-dilute concentration region (PA 1\%).

In summary, the symmetric electric field works asymmetrically on the kinks in a DNA chain through the entanglement effect between DNA and host polymers. Then, the kinks move only in the direction that the apexes point to, resulting in an increase of the end-to-end distance of DNA. The kinks can disappear only at the ends of the DNA chain. Consequently, this process induces the DNA-stretching.

\section{CONCLUSION}

We investigated the dynamics of a long DNA chain under AC electric fields in concentrated polymer solutions. Especially, the dependence of conformational size of $\lambda$ DNA on frequency was studied. We classified the frequency into four regions, each corresponding to linear motion, anti-resonance, stretching, and orienting of DNA. In the region of stretching, DNA molecules with the contour length ranging from $16 \mu \mathrm{m}$ to $200 \mu \mathrm{m}$ were elongated. The stretching process of T4 DNA was investigated in detail, and the importance of the asym- metric effect of the electric field was demonstrated. In concentrated polymer solutions, kinks larger than a certain size move only in the direction that the apexes point to. The asymmetric effect was explained from the tube model. We made a conjecture that the mechanism of stretching would be independent of the contour length of DNA. This conjecture remains to be proved for an extremely long DNA, such as Human genome chromosomal DNA.

Acknowledgments. We would like to thank the following persons, Prof. Hiroshi Iwasaki (ISIR, Osaka University), Ms. Sonia Antoranz Contera (ISIR, Osaka University), and Ms. Mariko Nakajima (NOV Architectural Atelier) for their helpful suggestions. This work is partially supported by a Grant-in-Aid for Scientific Research from the Ministry of Education, Science, Sports and Culture of Japan (No. 08226224), and Yamada Science Foundation.

\section{REFERENCES}

1. K. Morikawa and M. Yanagida, J. Biochem., 89, 693 (1981).

2. S. B. Smith, P. K. Aldridge, and J. B. Callis, Science, 243, 203 (1989).

3. D. C. Schwartz and M. Koval, Nature, 338, 520 (1989).

4. N. J. Rampino and A. Chrambach, Biopolymers, 31, 1297 (1991).

5. Y. Masubuchi, H. Oana, K. Ono, M. Matsumoto, M. Doi, K. Minagawa, Y. Matsuzawa, and K. Yoshikawa, Macromolecules, 26, 5269 (1993)

6. H. Oana, Y. Masubuchi, M. Matsumoto, M. Doi, Y. Matsuzawa, and K. Yoshikawa, Macromolecules, 27, 6061 (1994).

7. X. Shi, R. W. Hammond, and M. D. Morris, Anal. Chem., 67, 3219 (1995)

8. M. Ueda and Y. Baba, Anal. Sci., 13, 109 (1997).

9. M. Ueda, H. Oana, Y. Baba, M. Doi, and K. Yoshikawa, Biophys. Chem., 71, 111 (1998).

10. M. Ueda, K. Yoshikawa, and M. Doi, Polym. J., 29, 1040 (1997).

11. M. Ueda, Nucl. Acids Res., Symp. Ser., No. 39, 59 (1998).

12. T. A. Duke, Phys. Rev. Lett., 62, 2877 (1989).

13. Y. Masubuchi, H. Oana, M. Matsumoto, M. Doi, and K. Yoshikawa, Electrophoresis, 17, 1065 (1996)

14. S. J. Hubert, G. W. Slater, and J. L. Viovy, Macromolecules, 29, 1006 (1996)

15. C. Rivetti, M. Guthold, and C. Bustamante, J. Mol. Biol., 264, 919 (1996). 